\title{
Semiclassical transport theory of inhomogeneous systems
}

\author{
L. Sheng \\ Department of Physics, University of Hong Kong, Pokfulam Road, Hong Kong \\ and National Laboratory of Solid State Microstructure, Nanjing University, Nanjing 210093, People's Republic of China \\ Z. D. Wang \\ Department of Physics, University of Hong Kong, Pokfulam Road, Hong Kong \\ D. Y. Xing \\ Chinese Center of Advanced Science and Technology (World Laboratory) P.O. Box 8730, Beijing, People's Republic of China \\ and Department of Physics, Nanjing University, Nanjing, People's Republic of China \\ Jian-Xin Zhu \\ Department of Physics, University of Hong Kong, Pokfulam Road, Hong Kong
}

(Received 8 May 1995)

\begin{abstract}
Based on the probability-conserved Boltzmann equation, we develop a formal and general transport theory for the conductivity in inhomogeneous systems. In particular, we show that the local current density inside the sample can be expressed as a boundary value integral, so that the local electric field need not be calculated explicitly. The theory is first applied to multilayer systems and shown to recover the previous theory. More importantly, by including spin-dependent interface scattering and bulk scattering, we employ our theory successfully to account for the giant magnetoresistance in magnetic granular systems.
\end{abstract}

Magnetotransport in inhomogeneous magnetic structures, such as magnetic multilayers ${ }^{1}$ and magnetic granular solids, ${ }^{2,3}$ exhibits often a feature of very large and negative magnetoresistance (MR). This effect has attracted much attention in recent years. The physical interpretation of the giant MR effect is usually based on spin-dependent interface scattering as well as on spin-dependent bulk scattering. Semiclassical models $\mathrm{s}^{4-6}$ based on the Boltzmann equation and quantum theories ${ }^{7,8}$ starting from the Kubo formula have been proposed to account for the giant MR effects in multilayer structures, for currents being both in the plane (CIP) and perpendicular to the plane of the layers (CPP). On the other hand, theories of the MR in granular systems have not been quite well developed as the spatial distributions of the field and the currents are more complicated in these inhomogeneous systems.

In the earlier publications, ${ }^{2,9}$ it is assumed that the transport in granular systems is very closed to the CPP case in multilayers, which is equivalent to an assumption of a uniformly distributed current density. This approximation neglects the effect that the currents tend to bypass the obstacles, leading to an underestimation of the value of the conductivity. In this paper, based on a probability-conserved Boltzmann equation, we develop a transport theory of inhomogeneous systems with impurity scattering and interface scattering included. The theory is first applied to multilayers for the CIP and CPP cases and is found to recover the previous theory. For granular systems, within the mean-field approximation, an analytical expression for the MR is derived and the results obtained agree well with the experimental measurements on the MR effect.

Formal theory. Let us consider a general inhomogeneous system in which charge carriers scatter with impurities and rough interfaces. For convenience, we do not include the spin freedoms for a while. In the presence of an external electric field $\mathbf{E}^{\text {ex }}$, the Boltzmann equation describing this inhomogeneous system can be written as

$$
\mathbf{v} \cdot \nabla g+(g-\bar{g}) / \tau=e \mathbf{v} \cdot \mathbf{E},
$$

where $\tau$ is the relaxation time, $g$ is a function characterizing the deviation of the distribution function $f$ from the equilibrium distribution $f_{0}$, which satisfies the relation $f=f_{0}+g\left(\partial f_{0} / \partial \varepsilon\right), \quad$ and $\bar{g}(v, \mathbf{r})=(4 \pi)^{-1} \int d \Omega_{v} g(\mathbf{v}, \mathbf{r})$. $\mathbf{E}(\mathbf{r})$ is the actual electric field in the system. Since interface scattering is also considered as impurity scattering in thin mixing films, ${ }^{8}$ all of the scattering effect is included in the position-dependent relaxation time $\tau(\mathbf{r})$. Notice that, within this model, the continuity of the current $\nabla \cdot \mathbf{J}=0$ is automatically satisfied.

Equation (1) can be solved by the path integral approach, ${ }^{5}$ and the current density is obtained as

$$
\mathbf{J}(\mathbf{r})=C_{e} \int d^{3} r^{\prime} \hat{\mathbf{r}}_{0} \Phi\left(\mathbf{r}, \mathbf{r}^{\prime}\right)\left[\frac{\bar{g}\left(\mathbf{r}^{\prime}\right)}{e \lambda\left(\mathbf{r}^{\prime}\right)}+\hat{\mathbf{r}}_{0} \cdot \mathbf{E}\left(\mathbf{r}^{\prime}\right)\right],
$$

where $C_{e}=3 n_{e} e^{2} / 2 m v_{F}$ with $v_{F}$ the Fermi velocity and $m$ the effective mass, $\hat{\mathbf{r}}_{0}=\left(\mathbf{r}-\mathbf{r}^{\prime}\right) /\left|\mathbf{r}-\mathbf{r}^{\prime}\right|$, and

$$
\Phi\left(\mathbf{r}, \mathbf{r}^{\prime}\right)=\frac{1}{4 \pi\left|\mathbf{r}-\mathbf{r}^{\prime}\right|^{2}} \exp \left[-\int_{\mathbf{r}^{\prime} \rightarrow \mathbf{r}} \frac{d s^{\prime \prime}}{\lambda\left(\mathbf{r}^{\prime \prime}\right)}\right],
$$

with $\lambda(\mathbf{r})=v_{F} \tau(\mathbf{r})$ as the mean free path of carriers. The integral is along the straight line connecting the points $\mathbf{r}$ and $\mathbf{r}^{\prime}$, and $d s^{\prime \prime}$ is the element of the line segment at the point $\mathbf{r}^{\prime \prime} . \bar{g}(\mathbf{r})$ is in connection with the electric field via the relation 


$$
\bar{g}(\mathbf{r})=\int d^{3} r^{\prime} \Phi\left(\mathbf{r}, \mathbf{r}^{\prime}\right)\left[\frac{\bar{g}\left(\mathbf{r}^{\prime}\right)}{e \lambda\left(\mathbf{r}^{\prime}\right)}+\hat{\mathbf{r}}_{0} \cdot \mathbf{E}\left(\mathbf{r}^{\prime}\right)\right] .
$$

In the above expressions, the argument of velocity is omitted since it takes the constant value $v_{F}$. It is useful to define the two-point conductivity tensor $\sigma_{\alpha \beta}$ by

$$
J_{\alpha}(\mathbf{r})=\int d^{3} r^{\prime} \sigma_{\alpha \beta}\left(\mathbf{r}, \mathbf{r}^{\prime}\right) E_{\beta}\left(\mathbf{r}^{\prime}\right),
$$

where the summation is indicated by two same coordinate labels. It is convenient to employ the matrix representation and represent formally the integrals over coordinates by matrix multiplications. Equation (4) is first solved by iteration, and then by substituting the solution into Eq. (2), we arrive at a series expression for the two-point conductivity tensor:

$$
\sigma_{\alpha \beta}=\sigma_{\alpha \beta}^{c}+\sigma_{\alpha \beta}^{d},
$$

where $\quad \sigma_{\alpha \beta}^{c}\left(\mathbf{r}, \mathbf{r}^{\prime}\right)=C_{e} r_{0 \alpha} r_{0 \beta} \Phi\left(\mathbf{r}, \mathbf{r}^{\prime}\right) \quad$ and $\quad \sigma_{\alpha \beta}^{d}=$ $C_{e} D_{\alpha} \Lambda G D_{\beta}$, with

$$
G=(1-\Phi \Lambda)^{-1}=1+\Phi \Lambda+\cdots,
$$

$D_{\alpha}\left(\mathbf{r}, \mathbf{r}^{\prime}\right)=r_{0 \alpha} \Phi\left(\mathbf{r}, \mathbf{r}^{\prime}\right)$, and $\Lambda\left(\mathbf{r}, \mathbf{r}^{\prime}\right)=\delta\left(\mathbf{r}-\mathbf{r}^{\prime}\right) / \lambda(\mathbf{r})$.

In principle, the actual local electric field $\mathbf{E}(\mathbf{r})$ in Eq. (1) needs to be determined self-consistently from the spatially redistributed charge induced by the field itself, which is highly nontrivial. Fortunately, we can show it is unnecessary to calculate this field explicitly if we only attempt to calculate the conductivity. It is direct to find from Eq. (6) the relation

$$
\frac{\partial}{\partial r_{\alpha}} \sigma_{\alpha \beta}\left(\mathbf{r}, \mathbf{r}^{\prime}\right)=\frac{\partial}{\partial r_{\beta}^{\prime}} \sigma_{\alpha \beta}\left(\mathbf{r}, \mathbf{r}^{\prime}\right)=0,
$$

which is essentially a deduction of the continuity condition of currents. Now, we can transform the volume integral in Eq. (5) into the surface integral over the sample,

$$
J_{\alpha}(\mathbf{r})=\oint_{\text {sample }}\left(d S^{\prime}\right)_{\beta} \sigma_{\alpha \beta}\left(\mathbf{r}, \mathbf{r}^{\prime}\right) U\left(\mathbf{r}^{\prime}\right),
$$

where $U$ is the electric potential satisfying $\mathbf{E}=-\nabla U$ and $\left(d S^{\prime}\right)_{\beta}$ is the $\beta$ th component of the area element $d \mathbf{S}^{\prime}$. Here, we consider that the sample is linked to the reservoirs by ideal electrodes so that the field in the electrodes far from the sample is zero and its contribution to the current inside the sample vanishes. From Eq. (8), we reach a crucial conclusion that the local current density depends only on the electrical potential on the boundary, not the specific distribution of the electric field inside the sample, which is essentially similar to the results obtained by Landauer-Buettiker scattering theory. ${ }^{10}$ Its merit lies in the fact that, rather than solving the coupled problem of charge and field distributions selfconsistently, one can obtain the full spatial information from a two-point conductivity tensor. Therefore, in the calculation of the conductivity, one can choose the electric field in Eq. (8) [or Eq. (5)] with some freedom, as long as the boundary conditions are suitably considered. It is quite convenient to choose the applied uniform field $\mathbf{E}^{\mathrm{ex}}$ in the calculation as it could give the same boundary value integral as the actual field. Equation (5) becomes

$$
J_{\alpha}=\sigma_{\alpha \beta}^{c} E_{\beta}^{\mathrm{ex}}+C_{e} D_{\alpha} \Lambda \mu,
$$

with $\mu=G D_{\beta} E_{\beta}^{\mathrm{ex}}$. From Eq. (9) and by some algebra, we obtain

$$
J_{\alpha}(\mathbf{r})=\int d^{3} r^{\prime} \sigma_{\alpha \beta}^{c}\left(\mathbf{r}, \mathbf{r}^{\prime}\right) E_{\beta}^{\mathrm{eff}}\left(\mathbf{r}^{\prime}\right),
$$

where $\mathbf{E}^{\text {eff }}=\mathbf{E}^{\text {ex }}-\nabla \mu$. Equation (10) or Eq. (9) is the formal solution of the Boltzmann equation (1), which can be used in general cases and is a central result of this work. In this paper, it is applied to three cases: superlattices with CIP, superlattices with CPP, and magnetic granular solids.

It is worthwhile to mention that the above results can also be obtained by using an effective Boltzmann equation $\mathbf{v} \cdot \nabla g+g / \tau=e \mathbf{v} \cdot \mathbf{E}^{\text {eff }}$. Since this effective equation does not satisfy the continuity of currents, the effective electric field $\mathbf{E}^{\text {eff }}(\mathbf{r})$ should be determined from the continuity condition of currents, which is nontrivial. This equation could be considered to be a phenomenological version of our theory, with $\mathbf{E}^{\mathrm{eff}}(\mathbf{r})$ as the internal field.

Notice that there exist two conventional definitions of the average conductivity, i.e., $\langle J\rangle / E^{\mathrm{ex}}$ and $\langle J\rangle /\left\langle E^{\mathrm{eff}}\right\rangle$. For macroscopic systems, it is expected that they should be equivalent since the contact resistance is negligible when compared with the resistance of the sample. This point can be justified as follows. Multiplying Eq. (9) and Eq. (10) by $1 / \lambda(\mathbf{r})$ and taking the averages over the whole sample, we can find $\mathbf{E}^{\mathrm{ex}}=\left\langle\mathbf{E}^{\mathrm{eff}}\right\rangle$.

Application to superlattices. We first apply our general theory to investigate transport in superlattices. If the $z$ axis is chosen along the stacked direction of the layers, the twopoint conductivity is found to be diagonalized for the coordinate labels $(\alpha \beta)$. Due to the translation invariance, Eq. (10) reduces to the one-dimensional form

$$
J_{\alpha}(z)=\int d z^{\prime} \sigma_{\alpha \alpha}^{c}\left(z, z^{\prime}\right) E_{\alpha}^{\mathrm{eff}}\left(z^{\prime}\right) .
$$

The two-point conductivity is now given by $\sigma_{\alpha \alpha}^{c}\left(z, z^{\prime}\right)$ $=C_{e} F_{\alpha}(\xi) / 4$, where $\xi=\left|\int_{z^{\prime}}^{z} d z^{\prime \prime} / \lambda\left(z^{\prime \prime}\right)\right|$ and $F_{\alpha}(t)=E_{1}(t)$ $-E_{3}(t)$ for CIP and $F_{\alpha}(t)=2 E_{3}(t)$ for CPP with $E_{n}(t)$ the $n$th order exponential integral function. Since the applied electric field is simply a constant, the integral $\int d^{3} r^{\prime} D_{\alpha}\left(\mathbf{r}, \mathbf{r}^{\prime}\right) E_{\alpha}^{\mathrm{ex}}$ in $\mu$ is found to be zero in the case of CIP. Therefore, $E^{\text {eff }}=E^{\text {ex }}$ for CIP. The average conductivity is then $\sigma_{\|}=L^{-1} \int_{0}^{L} d z \int_{-\infty}^{\infty} \sigma_{x x}\left(z, z^{\prime}\right)$, with $L$ the period of the superlattice. For the case of CPP, one can verify directly that the effective field is $E^{\text {eff }}=E^{\mathrm{ex}} \bar{\lambda} / \lambda(z)$ with $\bar{\lambda}^{-1}=L^{-1} \int_{0}^{L} d z / \lambda(z)$. Consequently, the average conductivity is evaluated to be $\sigma_{\perp}=n e^{2} \bar{\lambda} / 2 k_{F}{ }^{6}{ }^{6}$ Those results previously obtained in Refs. 7 and 8 are recovered in the present general theory.

Magnetoresistance in magnetic granular systems. Let us turn to investigate the transport in granular systems within the framework of a mean-field treatment in our formal theory. We consider the $N_{p}$-particle system to be realized by adding one particle, which will be called the central particle, to the system of $N_{p}-1$ particles. The effective field in the system of $N_{p}-1$ particles is written as $\mathbf{E}^{\mathrm{bc}}$, which is essentially the sum of the applied field and the field produced by 
the accumulated charge on the $N_{p}-1$ particles. The $N_{p}$-particle problem is then treated roughly as that one of an isolated particle is in the background field $\mathbf{E}^{\mathrm{bc}}$.

With the help of our formal theory, the above considerations can be represented more concretely. From Eq. (7), we can write

$$
G_{N_{p}}=G_{N_{p}-1}+G_{N_{p}} V_{1} G_{N_{p}-1}
$$

and

$$
\begin{aligned}
V_{1} & =\Phi_{N_{p}} \Lambda_{N_{p}}-\Phi_{N_{p}-1} \Lambda_{N_{p}-1} \\
& \approx \Phi_{1} \Lambda_{1}-\Phi_{0} \Lambda_{0},
\end{aligned}
$$

where the subscript $N_{p}$ corresponds to $N_{p}$-particle system and the subscript 0 represents the bulk medium with no particle embedded. The second equality in Eq. (13) is a reasonable approximation in the low-density limits. By using Eq. (13), we obtain

$$
\begin{aligned}
\mu_{N_{p}}= & \mu_{N_{p}-1}+G_{N_{p}} V_{1} \mu_{N_{p}-1}+G_{N_{p}-1} D_{1, \beta} E_{\beta}^{\mathrm{ex}} \\
& +G_{N_{p}} V_{1} G_{N_{p}-1} D_{1, \beta} E_{\beta}^{\mathrm{ex}} .
\end{aligned}
$$

Since $V_{1}$ is localized in the small region of the central particle, we can neglect the interference of the other particles in the factor $G_{N_{p}}$ in the second term, and it thus becomes $G_{1} V_{1} \mu_{N_{p}-1}$. Similarly, we approximate the third and fourth terms in Eq. (14) as $G_{0} D_{1, \beta} E_{\beta}^{\mathrm{ex}}$ and $G_{1} V_{1} G_{0} D_{1, \beta} E_{\beta}^{\mathrm{ex}}$, respectively. Consequently, Eq. (14) is derived to be

$$
\mu_{N_{p}}=\mu_{N_{p}-1}+G_{1} D_{1, \beta} E_{N_{p}-1, \beta}^{\mathrm{eff}} .
$$

By taking gradient on the both sides of Eq. (15), we obtain

$$
\mathbf{E}^{\mathrm{eff}}=\mathbf{E}^{\mathrm{bc}}-\nabla\left(G_{1} D_{1, \beta} E_{\beta}^{\mathrm{bc}}\right),
$$

where $\mathbf{E}^{\mathrm{eff}}=\mathbf{E}_{N_{p}}^{\mathrm{eff}}$ and $\mathbf{E}^{\mathrm{bc}}=\mathbf{E}_{N_{p}-1}^{\mathrm{eff}}$. Equation (16) is highly useful for us to determine the effective electric field in the $N_{p}$-particle system, because we need only to solve the problem of an isolated particle in the background electric field. In the above calculations, we have used the approximation that the background field is uniform, which is reasonable in the small region of the central particle, and so Eq. (16) is appropriate only in this small region. Nevertheless, using this equation in the region of each particle, we are able to obtain the field in the whole system.

To obtain the conductivity analytically, we now take the local limits, i.e.,

$$
\sigma_{\alpha \beta}^{c}\left(\mathbf{r}, \mathbf{r}^{\prime}\right)=\sigma(\mathbf{r}) \delta_{\alpha \beta} \delta\left(\mathbf{r}-\mathbf{r}^{\prime}\right),
$$

where $\sigma(\mathbf{r})=n e^{2} \lambda(\mathbf{r}) / 2 m v_{F}$, which is reasonable when the mean free path is much less than the distance between the particles. In this case, since the essential transport of the system is determined mainly by the scattering at interfaces, in medium and inside particles, as usual, it is quite acceptable to take $\lambda(\mathbf{r})$ effectively as $\lambda_{I}, \lambda_{0}$, and $\lambda_{F} \cdot{ }^{6,8,11}$ Since $\nabla \times \mathbf{E}^{\mathrm{eff}}=0$, it is convenient to define a scalar potential by $\mathbf{E}^{\text {eff }}(\mathbf{r})=-\nabla U^{\mathrm{eff}}(\mathbf{r})$. Then continuity equation of currents becomes the Poisson's equation of the scalar potential in all regions. Associated with this equation, the boundary condition that let the effective field be the background field $\mathbf{E}^{\text {bc }}$ for $r \rightarrow \infty$ and the fixed potential drop between the ends of the sample must be considered. In fact, we need only to consider the former condition as the later can be included in the redefined background field. Solving Poisson's equation, we have

$$
U^{\mathrm{eff}}(r, \theta)=-\cos (\theta) \begin{cases}c d E^{\mathrm{bc}} r+D / r^{2}, & r>a+d, \\ C_{1} r+C_{2} / r^{2}, & a+d>r>a, \\ E^{F} r, & a>r,\end{cases}
$$

where $d$ and $a$ are, respectively, the thickness of the mixing film and the radius of the particle, $D, C_{1}, C_{2}$, and $E^{F}$ are constants to be determined from the continuity conditions at the two surfaces of the interlayer. In particular, as $d \ll a$, the effective field in the particle is

$$
E^{F}=\frac{3 \lambda_{0} E^{\mathrm{bc}}}{2 \lambda_{0}+\lambda_{F}+2 \lambda_{0} \lambda_{F} R_{I} / a},
$$

where $R_{I}=d / \lambda_{I}$. If we let $C_{e} R_{I} / 3=r_{s}$, we can find that Eq. (18) is the same as Eq. (7) in Ref. 11, apart from the difference of $E^{\mathrm{bc}}$ and $E$. The condition $C_{e} R_{I} / 3=r_{s}$ links the two phenomenological parameters characterizing the interface scattering in two different models: A interface is modeled by a mixing film, and characterized by a resistance that leads to voltage drop between the two sides. However $E^{\text {bc }}$ cannot be replaced by the applied field; otherwise, unphysical results such as positive MR (Ref. 12) in Ref. 11 may be obtained.

We proceed to include the boundary condition of a fixed voltage drop. In fact, the field satisfying the boundary condition is the sums of the field of the charge on the particle and the field produced by a series of properly arranged imaginary charges outside the sample. Since the imaginary charge is very far from the central particle, its field is uniform in the small region of the central particle. Therefore, the boundary condition of the fixed voltage drop can be included simply by redefining the background field $\mathbf{E}^{\mathrm{bc}}$ to include the field of the imaginary charge.

Now we need to calculate the average of the effective electric field. We emphasize that even when the thickness goes to zero, the contribution from the field in the interlayer cannot be neglected. Considering that the field in the particle is a uniform value $E^{F}$, and is a dipolar one outside which is included in the background field of other particles, we obtain the relation determining $E^{\mathrm{bc}}$ :

$$
E^{\mathrm{ex}}=\left[(1-f)+\left(\gamma_{F}+\gamma_{I}\right) f\right] E^{\mathrm{bc}},
$$

where $f$ is volumetric filling factor, $\gamma_{F}=E^{F} / E^{\mathrm{bc}}$, and $\gamma_{I}=2 R_{I} \gamma_{F} \lambda_{F} / a$. The average conductivity is evaluated from $\langle J\rangle / E^{\mathrm{ex}}$ :

$$
\frac{\sigma}{\sigma_{0}}=\frac{(1-f)+f \gamma_{F} \lambda_{F} / \lambda_{0}}{2(1-f)+2\left(\gamma_{F}+\gamma_{I}\right) f},
$$

with $\sigma_{0}=n e^{2} \lambda_{0} / m v_{F}$.

In order to account for the giant MR effect, the spin freedoms should be included. We focus on the cases where the spin diffusion length is much larger than the mean free path, so that the total conductivity is the sum from the two spin channels and for each channel the previous formulas can be extended straightforwardly. If we let $\lambda_{I}^{\uparrow(\downarrow)}$ and $\lambda_{F}^{\uparrow(\downarrow)}$ denote 


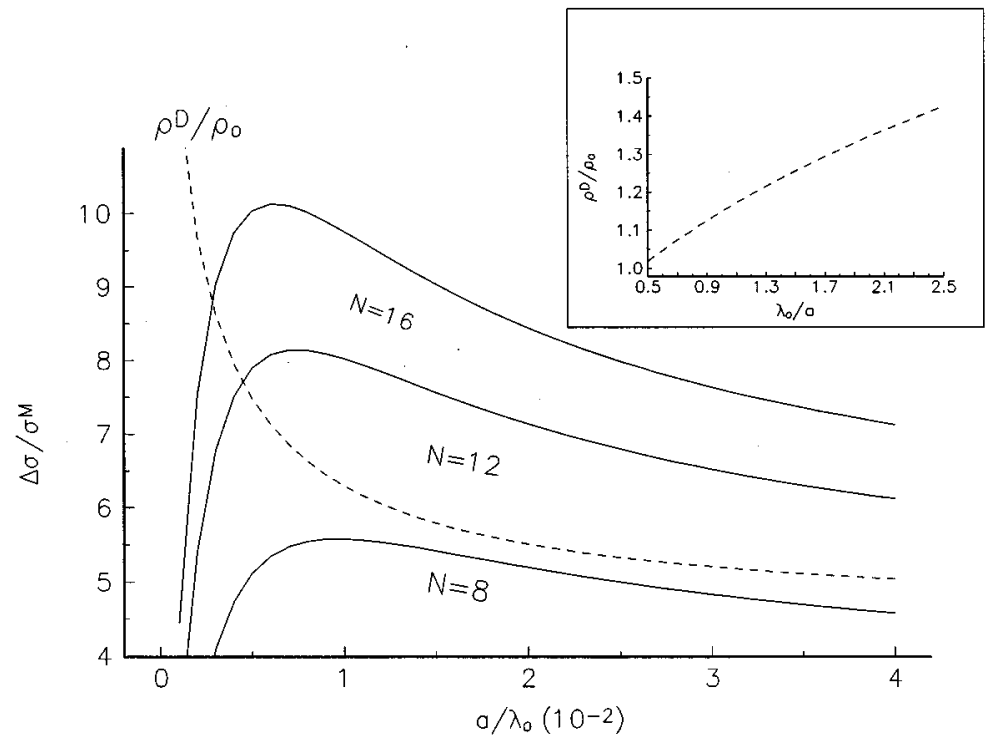

FIG. 1. Percent magnetoresistance $\Delta \sigma / \sigma^{M}$ versus $a / \lambda_{0}$ for three different spin-asymmetric factors $N=\lambda_{F}^{\downarrow} / \lambda_{F}^{\uparrow}=R_{I}^{\uparrow} / R_{I}^{\downarrow}$ (solid lines). The behavior of the zero-magnetic-field resistivity $\rho^{D} / \rho_{0}=\sigma_{0} / \sigma^{D}$ for $N=12$ is also shown via the relation $\left[6\left(\rho^{D} / \rho_{0}\right)-0.8\right]$ (dashed line) for clarity. The resistivity against the inverse of the particle size is illustrated in the upper panel. The other parameters are $f=0.3, \lambda_{F}^{\uparrow}=0.8 \lambda_{0}$, and $R_{I}^{\uparrow}=1.0$.

the mean free path of electrons with majority (minority) $\operatorname{spin}^{4}$ in the mixing films and in the particles, respectively, we obtain

$$
\frac{\sigma^{D}}{\sigma_{0}}=\frac{2(1-f)+f\left(\gamma_{F}^{\uparrow} \lambda_{F}^{\uparrow}+\gamma_{F}^{\downarrow} \lambda_{F}^{\downarrow}\right) / \lambda_{0}}{2(1-f)+\left(\gamma_{F}^{\uparrow}+\gamma_{F}^{\downarrow}+\gamma_{I}^{\uparrow}+\gamma_{I}^{\downarrow}\right) f}
$$

for the demagnetized state and

$$
\frac{\sigma^{M}}{\sigma_{0}}=\frac{1}{2} \sum_{s=\uparrow, \downarrow} \frac{(1-f)+f \gamma_{F}^{s} \lambda_{F}^{s} / \lambda_{0}}{(1-f)+\left(\gamma_{F}^{s}+\gamma_{I}^{s}\right) f}
$$

for the magnetized state. From Eqs. (21) and (22), $\Delta \sigma=\sigma^{M}-\sigma^{D}$ is found to be always positive, which corresponds to a negative MR.

In Fig. 1, we plot the MR amplitude as a function of the radius. The spin-dependent scattering in the particles and in the mixing films is considered to come from the same mechanism, ${ }^{7}$ and so we assume that the spin-asymmetric factor $\lambda_{I}^{\downarrow} / \lambda_{I}^{\uparrow}$ in the particles equals $R_{I}^{\uparrow} / R_{I}^{\downarrow}$ in the mixing film. From Fig. 1 we find that the MR first increases and then decreases with increasing the particle size, and a maximum occurs in the middle region. As seen from the experimental observations, ${ }^{2}$ there is always an optimum annealing temperature in preparing the magnetic granular sample for MR. In view of the fact that the sizes of the particles are always increased with increasing the annealing temperature, ${ }^{3}$ our results are in good agreement with these observations. Notice that, as observed by experiments, ${ }^{3}$ the resistivity itself given by our theory (dashed line) is also a strong function of the radius (or annealing temperature), and varies inversely with particle size. Particularly, as shown in the upper panel of Fig. 1 , the dependence of the resistivity on the inverse of the radius is approximated to be linear in a broaden region. This result is consistent with both quantum theory ${ }^{9}$ and the experimental data. ${ }^{3}$

In summary, we have presented an efficient approach to calculate the conductivity in inhomogeneous systems based on the Boltzmann equation, with which the complicated computation in determining the actual electric field in the system can be skipped. In particular, a formal and general semiclassical transport theory is developed and has been successfully applied to superlattices with CIP and CPP. Within a mean-field framework the theory is also used to calculate the giant MR effect in magnetic granular systems, and the obtained results are in good agreement with experimental observations. Moreover, it is expected that our formal theory can be widely employed to investigate the transport properties in other inhomogeneous systems.

This work was supported by the RGC research grant of Hong Kong under No. HKU 262/95P, a CRCG grant at the University of Hong Kong, in part by a joint grant of the NSFC and NAMCC, and in part by the National Natural Science Foundation of China.
${ }^{1}$ X. Baibich et al., Phys. Rev. Lett. 61, 2472 (1988).

${ }^{2}$ X. Berkowitz et al., Phys. Rev. Lett. 68, 3745 (1992); J.Q. Xiao, J.S. Jiang, and C.L. Chien, ibid. 68, 3749 (1992).

${ }^{3}$ Peng Xiong, Gang Xiao, and J.Q. Wang, Phys. Rev. Lett. 69, 3220 (1992); Jian-Qing Wang and Gang Xiao, Phys. Rev. B 49, 3982 (1994).

${ }^{4}$ R.E. Camley and J. Barnas, Phys. Rev. Lett. 63, 664 (1989); T. Valvet and A. Fert, Phys. Rev. B 48, 7099 (1993).

${ }^{5}$ L. Sheng and D.Y. Xing, Phys. Rev. B 50, 1001 (1994); M. Liu and D.Y. Xing, ibid. 47, 12272 (1993).
${ }^{6}$ L. Sheng and D.Y. Xing, J. Phys. Condens. Matter 6, 7248 (1994).

${ }^{7}$ P.M. Levy, S. Zhang, and A. Fert, Phys. Rev. Lett. 65, 1643 (1990).

${ }^{8}$ H.E. Camblong and P.M. Levy, Phys. Rev. Lett. 69, 2835 (1992).

${ }^{9}$ S. Zhang and P.M. Levy, J. Appl. Phys. 73, 5315 (1993).

${ }^{10}$ X. Baranger and X. Stone, Phys. Rev. B 40, 8169 (1989).

${ }^{11}$ Mark Rubinstein, Phys. Rev. B 50, 3830 (1994).

${ }^{12}$ Note that Ref. 11 in fact defines $\Delta \sigma$ as $\sigma_{\text {eff }}^{M}-\sigma_{\text {eff }}^{D}$, and find that $\Delta \sigma<0$ from Eqs. (7), (9), and (10) in that reference. 